From The University of Texas M. D. Anderson Cancer Center, Houston, TX

Submitted January 29, 2008; accepted July 9, 2008; published online ahead of print at www.jco.org on October 27, 2008

Presented in part at the 32nd Annual Oncology Nursing Congress, April 24-27, 2007, Las Vegas, NV

Authors' disclosures of potential conflicts of interest and author contributions are found at the end of this article.

Corresponding author: Elihu Estey, MD University of Washington Medical Center, Fred Hutchinson Cancer Research Institute, Seattle Cancer Care Alliance, 825 Eastlake Ave E, G3-200, Seattle WA 98109; e-mail: eestey@u washington.edu.

(C) 2008 by American Society of Clinical Oncology

0732-183X/08/2635-5684/\$20.00 DOI: $10.1200 / J C O .2008 .16 .468$

\title{
Randomized Comparison of Cooked and Noncooked Diets in Patients Undergoing Remission Induction Therapy for Acute Myeloid Leukemia
}

Alison Gardner, Gloria Mattiuzzi, Stefan Faderl, Gautam Borthakur, Guillermo Garcia-Manero, Sherry Pierce, Mark Brandt, and Elihu Estey

\section{$\begin{array}{llllllll}\text { A } & \text { B } & \text { S } & \text { T } & \text { R } & \text { A } & \text { C } & \text { T }\end{array}$}

\section{Purpose}

A neutropenic diet is often used to prevent infection in patients with acute myeloid leukemia $(A M L)$. Although such a diet potentially entails inconvenience, its value is uncertain.

\section{Patients and Methods}

One hundred fifty-three patients admitted to a high-efficiency particulate air-filtered room (protected environment [PE]) to receive induction therapy for newly diagnosed AML were randomly assigned to a diet containing no raw fruits or vegetables (cooked diet) or to a diet containing fresh fruit and fresh vegetables (raw diet). Stratification was based on the patients' early risk of mortality (ERM) score. All patients received antibacterial and antifungal prophylaxis and remained on study until they were discharged from the PE. The outcomes of principal interest were major infection (pneumonia, bacteremia, or fungemia) and death; if the true probability of either event was $20 \%$ on the cooked arm and $40 \%$ on the raw arm, then the probability that the cooked arm would be selected as superior was $83 \%$.

\section{Results}

Seventy-eight patients were randomly assigned to the cooked arm, and 75 were assigned to the raw arm. The two groups were similar with respect to age, ERM, chemotherapy received, and days at risk. Twenty-nine percent of patients in the cooked group and 35\% of patients in the raw group developed a major infection $(P=.60)$. Time to major infection and survival time were similar in the two groups. Fever of unknown origin occurred in $51 \%$ of the cooked group and $36 \%$ of the raw group.

\section{Conclusion}

In patients treated in a PE, a neutropenic diet did not prevent major infection or death.

J Clin Oncol 26:5684-5688. (C) 2008 by American Society of Clinical Oncology

\section{INTRODUCTION}

It is well known that neutropenia predisposes patients to infection. ${ }^{1}$ It is similarly established that foods, particularly fresh fruits and vegetables, contain Escherichia coli, Pseudomonas aeruginosa, and other Gram-negative bacilli that can cause lifethreatening sepsis and pneumonia. ${ }^{2,3}$ These two facts have led to the use of the neutropenic diet. After querying 400 hospitals associated with the Association of Community Cancer Centers, Smith and Besser $^{4}$ reported that $78 \%$ of the 156 responding hospitals used such a diet, typically once neutropenia $(<1,000$ or $1,500 / \mu \mathrm{L})$ was documented. A wide range of dietary exclusions fell under the rubric of a neutropenic diet. However, $98 \%$ of such diets restricted fresh vegetables, and 93\% restricted fresh fruits and juices.
Despite the routine use of a neutropenic diet, there seems to be little evidence of its benefit. ${ }^{5}$ In contrast, data exist that demonstrate that at least some patients would prefer not to be limited to a neutropenic diet. Specifically, DeMille et $\mathrm{al}^{6}$ found that $30 \%$ of the 23 outpatients participating in a study of the effect of this diet on infection rate were not compliant with the mandates of the diet. Infection rates were similar in compliant and noncompliant patients. A small trial (19 patients total) in children (median age, 4 to 5 years) found no difference in rates of febrile neutropenia according to whether patients were randomly assigned to a US Food and Drug Administration-approved food safety diet or a diet that, in addition, did not allow raw fruits and vegetables, take-out food, or fast food. ${ }^{7}$ Similarly a small randomized trial (total of 20 patients, 15 with acute myeloid leukemia [AML]) 
found no differences in gut colonization or infection according to whether a normal hospital diet or a low bacterial diet was used. ${ }^{8}$ In each of these trials, it was unclear whether the different dietary groups were at equal underlying risk of infection.

To assess whether the benefits of a neutropenic diet justify its inconvenience, we performed a larger trial that randomly assigned patients with untreated AML or high-risk myelodysplastic syndrome (MDS) who were about to receive chemotherapy in highefficiency particulate air-filtered rooms (protected environment $[\mathrm{PE}])$ to either a diet that contained fruits and vegetables only if cooked, as per standard practice of The University of Texas M. D. Anderson Cancer Center, or to a diet that permitted fresh (ie, raw) fruits and vegetables.

\section{PATIENTS AND METHODS}

Patients admitted to the PE were eligible provided that, at the time of random assignment, they had neither pneumonia nor bacteremia and were about to receive remission induction therapy on an ongoing Leukemia Department protocol for AML or high-risk MDS (10\% to $19 \%$ blasts in marrow or blood). Two hundred six patients were eligible. Fifty-three of the patients $(26 \%)$ preferred to conform to the hospital's policy of eating a diet containing no raw fruits and vegetables. The remaining 153 patients consented to be randomly assigned to either the hospital's diet of eating no raw fruits or vegetables or a diet containing fresh fruits and vegetables. The Leukemia Department Data Management Office performed the random assignment using the patients' early risk of mortality (ERM) score ${ }^{9}$ as a stratification factor. The ERM incorporates pretreatment values for performance status, bilirubin, age, albumin, fibrinogen, absolute neutrophil count, hemoglobin, and creatinine to estimate the probability of death within 4 weeks after initiation of chemotherapy. The 604 patients admitted to the PE from 2000 to 2004 had a median ERM score of 0.17 (corresponding to $17 \%$ probability of death in the 28 days after beginning chemotherapy), leading us to stratify patients according to whether their ERM was greater or less than this value. Patients randomly assigned to the diet allowing fresh fruits and vegetables were encouraged to eat at least one of these daily, with the fruits and vegetables washed with cold water for 30 seconds before eaten. Compliance with the assigned diet was facilitated by placing notices of this diet on patients' charts and by use of diaries in which patients recorded what they ate each day. All patients remained on the correct diet while on study, although some did not eat a fresh fruit or vegetable every day as suggested.

All patients had central lines placed and received prophylaxis with levofloxacin, valacyclovir, and depending on protocol, itraconazole, voriconazole, or a lipid preparation of amphotericin B. If fever of unknown origin (FUO) or pneumonia occurred, patients received intravenous ceftazidime or equivalent; if fever did not resolve, antifungal coverage was broadened. Patients remained on study until they were discharged from the PE to the outpatient setting, usually after return of the neutrophil count to more than $500 / \mu \mathrm{L}$ or after 6 weeks in patients in whom neutrophil recovery was delayed. Granulocyte colony-stimulating factor was used only when there was a delay (eg, 6 weeks in neutrophil recovery or after a major infection developed), and its use was equally infrequent in the cooked and raw diet groups.

The principal outcomes of interest were major infection (pneumonia, bacteremia, or fungemia, or pneumonia accompanied by bacteremia or fungemia) and death; we also noted the rates of minor infections and FUO. The principal comparison was between the group randomly assigned to eat only cooked food and the group randomly assigned to eat fresh fruits and vegetables; these groups are hereafter referred to as cooked and raw, respectively. To allow the reader to evaluate whether the patients randomly assigned to the cooked group were representative, we also report the incidences in the nonrandomized group (ate only cooked food). A diagnosis of pneumonia required a compatible chest x-ray or computed tomography scan. Bronchoalveolar lavage to isolate a causative organism was performed if no resolution had occurred after 3 to 5 days. A diagnosis of bacteremia as a result of frequent contaminants such as coagulase-negative Staphylococcus required two positive blood cultures.

The statistical design was the Bayesian multiple outcome design of Thall et $\mathrm{al}^{10}$ as extended by Thall and Sung. ${ }^{11}$ The outcomes monitored were major infection and death. Specifically, if after every 20 patients were randomly assigned, the probability was more than 0.995 that the incidence of death or major infection was greater in either the raw or cooked arm, then the study would stop. Otherwise, 188 patients would be randomly assigned, with 94 patients on each arm. If the true probability of major infection was $20 \%$ on the cooked arm and $40 \%$ on the raw arm, then the probability that the cooked arm would be selected as superior at the end of the trial was $83 \%$; this is analogous to a power of $83 \%$ using a more traditional design. ${ }^{12}$ In contrast, if the true probability of major infection was $30 \%$ on both arms, then the probability that an arm would be selected as superior was 0.07 , corresponding to $P=.07$ in more traditional designs. ${ }^{12}$

We used the $\chi^{2}$ or Kruskal-Wallis test to compare various pretreatment characteristics; number of days on study and number of days with neutrophil counts less than $500 / \mu \mathrm{L}$ and less than $100 / \mu \mathrm{L}$; and rates of diarrhea, mucositis, and infection in the cooked, raw, and nonrandomized groups. Time to major infection and time to death in the cooked and raw groups were compared using the log-rank test. We performed approximately 40 tests of statistical significance. Although a standard Bonferroni correction, which would regard a $P$ value as significant if less than approximately $.001(0.05 / 40)$, is perhaps overly conservative given the related nature of many of the tests (eg, patients with any infection, patients with any infection or FUO), we would certainly advise regarding any $P>.01$ with caution. The M. D. Anderson Cancer Center Institutional Review Board approved this study, which was conducted in accordance with the Declaration of Helsinki.

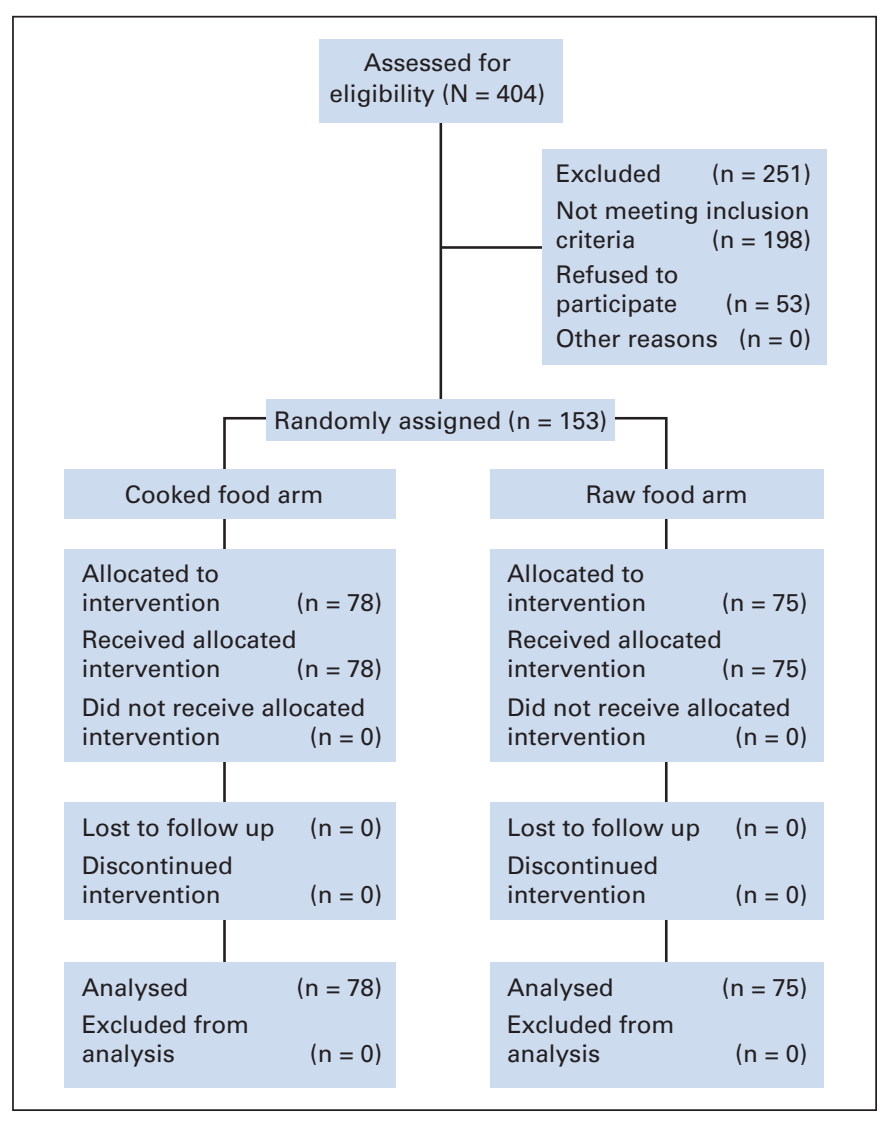

Fig 1. Flow of patients admitted to protected environment. 


\section{RESULTS}

Seventy-eight patients were randomly assigned to the cooked arm, and 75 were assigned to the raw arm; 53 patients chose not to be randomly assigned, thus eating only cooked food (Fig 1). The three groups were similar with respect to age and ERM score (Table 1). Patients in the cooked and raw groups received prophylaxis with voriconazole more often than non-randomly assigned patients, of note because we have reported that such prophylaxis is more effective than itraconazole or lipid amphotericin B in preventing fungal infection ${ }^{13}$; however, similar proportions of patients in the cooked and raw groups received voriconazole (Table 1). Patients in the cooked group were more likely than those in the raw group to receive cytarabine, but the two groups were equally likely to receive high-dose therapy of any kind as opposed to targeted therapy (eg, decitabine; Table 1). Patients were on study (ie, at risk) for 24 to 25 days in each group. Fifty-three percent of patients in the cooked group and $57 \%$ of patients in the raw group had an antecedent hematologic disorder. The number of days spent with neutrophil counts less than $500 / \mu \mathrm{L}$ and less than $100 / \mu \mathrm{L}$ were similar in the cooked and raw groups (median of 20 and 21 days at $<500 / \mu \mathrm{L}$ in the cooked and raw groups, respectively; $P=.77$; and median of 15 and 16 days at $<100 / \mu \mathrm{L}$ in the cooked and raw groups, respectively; $P=.53$ ). Twenty-eight patients in both the cooked and raw groups had lost weight (a median of $5 \mathrm{lb}$ in each group) when they came off study. The incidence of grade 3 to 4 mucositis or diarrhea was similar in the cooked and raw groups ( $10 \%$ and $16 \%$, respectively; $P=.34$ ), further suggesting that the anti-AML therapies were equitoxic in the two groups. Grade 3 to 4 diarrhea or mucositis was seen in only one of the 36 cooked or raw group patients not administered cytarabine compared with 19 of the 117 cooked or raw group patients administered cytarabine.

\section{Major Infection}

Twenty-nine percent and 35\% of patients in the cooked and raw groups, respectively, developed a major infection $(P=.60$; Table 2). An exact $95 \%$ CI for the true difference in rates is $-11 \%$ to $21 \%$. The rate of major infection in the nonrandomized group $(36 \%)$ was similar to the rate in the randomized cooked group. Time to development of a major infection was likewise similar in the three groups (Fig 2). Excluding coagulase-negative Staphylococcus and $\alpha$-hemolytic Streptococcus, the rates of major infection were $27 \%$ in the cooked group and $24 \%$ in the raw group $(P=.71)$; when excluding patients administered monotherapy with clofarabine or VNP40101M (Cloretazine; Vion Pharmaceuticals, New Haven, CT), major infections occurred in $22(31 \%)$ of 72 and $21(34 \%)$ of 61 patients in the cooked and raw groups, respectively $(P=.71)$.

Bearing in mind the multiple tests of statistical significance, the incidence of pneumonia was higher in the cooked group, whereas the

Table 1. Patient Characteristics

\begin{tabular}{|c|c|c|c|c|c|c|c|}
\hline \multirow[b]{2}{*}{ Characteristic } & \multicolumn{2}{|c|}{$\begin{array}{l}\text { Patients Randomly } \\
\text { Assigned to No } \\
\text { Fresh Fruits and } \\
\text { Vegetables (cooked } \\
\text { diet; } n=78 \text { ) }\end{array}$} & \multicolumn{2}{|c|}{$\begin{array}{l}\text { Patients Randomly } \\
\text { Assigned to Fresh } \\
\text { Fruits and } \\
\text { Vegetables (raw } \\
\text { diet; } \mathrm{n}=75 \text { ) }\end{array}$} & \multicolumn{2}{|c|}{$\begin{array}{l}\text { Patients Not } \\
\text { Randomly Assigned } \\
\text { (preferred cooked } \\
\text { diet; } n=53 \text { ) }\end{array}$} & \multirow[b]{2}{*}{$P$} \\
\hline & No. & $\%$ & No. & $\%$ & No. & $\%$ & \\
\hline Age, years & & & & & & & .62 \\
\hline Median & \multicolumn{2}{|c|}{64} & \multicolumn{2}{|c|}{63} & \multicolumn{2}{|c|}{66} & \\
\hline Range & \multicolumn{2}{|c|}{$17-88$} & \multicolumn{2}{|c|}{$47-84$} & \multicolumn{2}{|c|}{$49-81$} & \\
\hline \multicolumn{8}{|l|}{ ERM score } \\
\hline 25th percentile & \multicolumn{2}{|c|}{0.05} & \multicolumn{2}{|c|}{0.05} & \multicolumn{2}{|c|}{0.05} & .98 \\
\hline Median & \multicolumn{2}{|c|}{0.11} & \multicolumn{2}{|c|}{0.14} & \multicolumn{2}{|c|}{0.12} & \\
\hline 75th percentile & \multicolumn{2}{|c|}{0.25} & \multicolumn{2}{|c|}{0.26} & \multicolumn{2}{|c|}{0.32} & \\
\hline Disease & & & & & & & .63 \\
\hline AML & 75 & & 69 & & 51 & & \\
\hline MDS & 3 & & 6 & & 2 & & \\
\hline Voriconazole prophylaxis & 14 & 18 & 8 & 11 & 0 & & $.005^{*}$ \\
\hline \multicolumn{8}{|l|}{ Chemotherapy } \\
\hline With cytarabine & 65 & 83 & 52 & 69 & 40 & 75 & $.13 \dagger$ \\
\hline Idarubicin + cytarabine \pm tipifarnib & 32 & & 24 & & 21 & & \\
\hline Clofarabine + cytarabine & 20 & & 18 & & 16 & & \\
\hline Fludarabine + cytarabine & 10 & & 9 & & 3 & & \\
\hline Without cytarabine & 13 & 17 & 23 & 31 & 13 & 25 & \\
\hline Clofarabine & 3 & & 11 & & 2 & & \\
\hline VNP40101M & 4 & & 8 & & 8 & & \\
\hline High dose & 70 & 90 & 70 & 93 & 50 & 94 & .64 \\
\hline Targeted & 8 & 10 & 5 & 7 & 3 & 6 & \\
\hline No. of days on study & & & & & & & .27 \\
\hline Median & & & & & & & \\
\hline Range & & & & & & & \\
\hline
\end{tabular}




\begin{tabular}{|c|c|c|c|c|c|c|c|}
\hline \multirow[b]{2}{*}{ Infection and FUO } & \multicolumn{2}{|c|}{$\begin{array}{c}\text { Patients } \\
\text { Randomly } \\
\text { Assigned to No } \\
\text { Fresh Fruits and } \\
\text { Vegetables } \\
\text { (cooked diet; } \\
\mathrm{n}=78 \text { ) }\end{array}$} & \multicolumn{2}{|c|}{$\begin{array}{l}\text { Patients } \\
\text { Randomly } \\
\text { Assigned to } \\
\text { Fresh Fruits and } \\
\text { Vegetables } \\
\text { (raw diet; } \\
\mathrm{n}=75 \text { ) }\end{array}$} & \multirow[b]{2}{*}{$P($ cooked $v$ raw $)$} & \multicolumn{2}{|c|}{$\begin{array}{l}\text { Patients Not } \\
\text { Randomly } \\
\text { Assigned } \\
\text { (preferred } \\
\text { cooked diet; } \\
n=53 \text { ) }\end{array}$} \\
\hline & No. & $\%$ & No. & $\%$ & & No. & $\%$ \\
\hline Patients with any major infection & 23 & 29 & 26 & 35 & .60 & 19 & 36 \\
\hline Patients with pneumonia & 12 & 15 & 4 & 5 & .06 & 12 & 23 \\
\hline Patients with bacteremia or fungemia & 7 & 9 & 17 & 23 & .03 & 4 & 8 \\
\hline $\begin{array}{l}\text { Patients with pneumonia accompanied by } \\
\text { bacteremia or fungemia }\end{array}$ & 4 & 5 & 5 & 7 & .74 & 3 & 6 \\
\hline Patients with any minor infection & 5 & 6 & 4 & 5 & .99 & 4 & 8 \\
\hline Patients with FUO & 40 & 51 & 27 & 36 & .07 & 22 & 42 \\
\hline Patients with either major or minor infection & 28 & 36 & 30 & 40 & .62 & 23 & 43 \\
\hline Patients with infection or FUO & 68 & 87 & 57 & 76 & .09 & 45 & 85 \\
\hline
\end{tabular}

Abbreviation: FUO, fever of unknown origin.

incidence of bacteremia was higher in the raw group. With either type of infection, the nonrandomized group bore more resemblance to the cooked group than the raw group. In only one of the 28 patients with pneumonia unaccompanied by bacteremia or fungemia was an organism recovered (Aspergillus species in a patient in the cooked group), and there were only three other documented fungal infections (one in each of the cooked, raw, and nonrandomized groups; Table 3 ). Organisms resident in the gut (E coli, Enterococcus, Candida albicans, Enterobacter, Pseudomonas, and Klebsiella) were cultured from blood in $6 \%$ of the cooked group and $15 \%$ of the raw group $(P=.12$; exact $95 \% \mathrm{CI},-.04$ to .23$)$.

\section{Death}

There was no suggestion that patients randomly assigned to the cooked group lived longer than those assigned to the raw group (Fig 3). Survival in all three groups was as expected in newly diagnosed AML or MDS patients, as were complete response rates $56 \%$ in

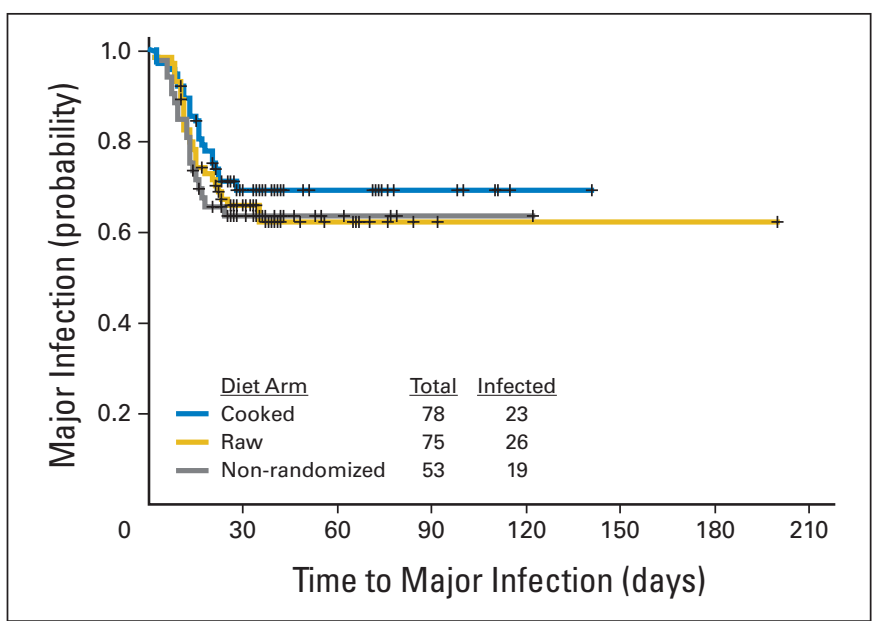

Fig 2. Probability of major infection in cooked, raw, and nonrandomized groups; the nonrandomized group ate only cooked food (log-rank test, $P=.50$ for three-way comparison and $P=.44$ for comparison of cooked and raw groups). cooked group, $61 \%$ in raw group, and $64 \%$ in patients not randomly assigned).

\section{Minor Infection and FUO}

Minor infections were observed in less than $10 \%$ of patients in all three groups. FUO was more common in the cooked group than the raw group. As typically occurs in treatment of AML or high-risk MDS, the great majority of patients had either an infection or an FUO $(87 \%$ in cooked group, $76 \%$ in raw group, and $85 \%$ in patients not randomly assigned; Table 2).

\begin{tabular}{|c|c|c|c|}
\hline \multirow[b]{2}{*}{ Organism } & \multicolumn{3}{|c|}{ No. of Patients } \\
\hline & $\begin{array}{l}\text { Cooked } \\
\text { Group }\end{array}$ & $\begin{array}{l}\text { Raw } \\
\text { Group }\end{array}$ & $\begin{array}{c}\text { Nonrandomized } \\
\text { Group }\end{array}$ \\
\hline \multicolumn{4}{|l|}{ Pneumonia } \\
\hline Aspergillus & 1 & - & - \\
\hline Unknown & 11 & 4 & 12 \\
\hline \multicolumn{4}{|l|}{ Bacteremia/fungemia \pm pneumonia } \\
\hline Escherichia coli & 2 & 3 & - \\
\hline Enterococcus & 2 & $5^{*}$ & 1 \\
\hline Staphylococcus aureus & 1 & - & - \\
\hline Coagulase-negative Staphylococcus & 1 & 3 & 1 \\
\hline$\alpha$-Hemolytic Streptococcus & 1 & 5 & - \\
\hline Enterobacter & - & 1 & 1 \\
\hline Neisseria & 1 & - & - \\
\hline Pseudomonas & - & 1 & 1 \\
\hline Klebsiella & - & 1 & 1 \\
\hline Stomatococcus & - & - & 1 \\
\hline Flavimonas & 1 & - & - \\
\hline Scedosporium & - & - & 1 \\
\hline Alcaligenes xylosoxidans & 1 & - & - \\
\hline Candida albicans & 1 & - & - \\
\hline Corynebacterium & - & 1 & - \\
\hline Roseomonas & - & 1 & - \\
\hline Capnocytophaga & - & 1 & - \\
\hline Fusarium & - & $1^{*}$ & - \\
\hline
\end{tabular}




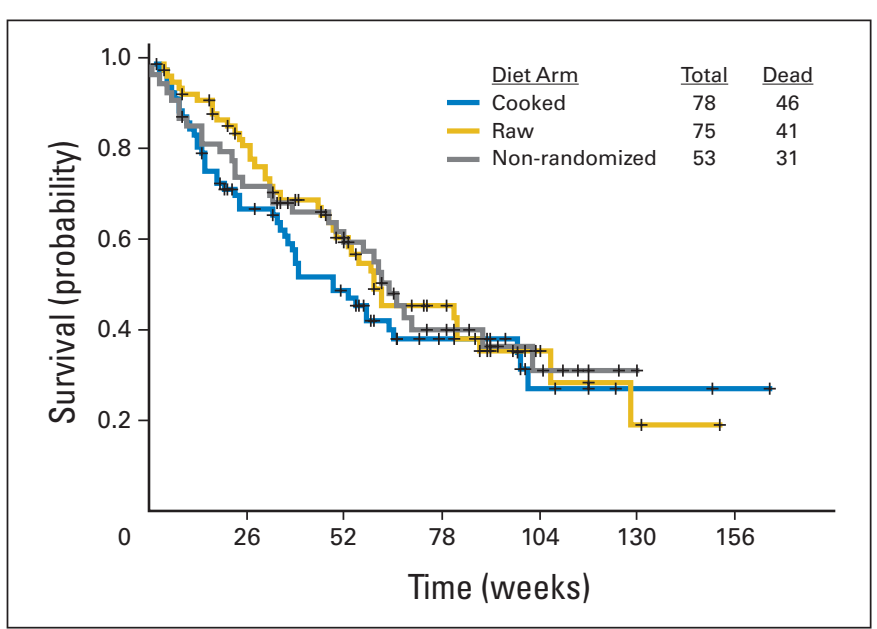

Fig 3. Probability of death in cooked, raw, and nonrandomized groups (log-rank test, $P=.32$ for three-way comparison and $P=.36$ for comparison of cooked and raw groups).

\section{DISCUSSION}

Our results suggest that there is little to be gained from use of a neutropenic diet in patients undergoing remission induction therapy for newly diagnosed AML or high-risk MDS. In particular, rates of major infection and death, the two major end points, were similar regardless of whether patients were randomly assigned to eat a cooked or raw diet. The exact $95 \%$ CI for the difference in rates of major infection suggests that a difference of no more than $10 \%$ is as plausible as no difference because 0 and 0.10 are equidistant from the midpoint of the CI (0.05), whereas the probability of survival was slightly higher in the raw group, making it unlikely that death would in fact have been more frequent in this group than the cooked group had more patients been entered. Although the incidence of bacteremia was higher in the raw group, a substantial part of this difference reflected isolation of organisms not resident in the gut; the presence of such organisms would not be expected to be influenced by cooking of fruits and vegetables. Furthermore, the incidence of FUO and, hence, of potentially false-negative bacteremias was higher in the cooked group.

A major question is the extent to which our results can be generalized. This question arises because $26 \%$ of patients who were eligible for random assignment declined to be randomly assigned (preferring to eat only cooked food). The observation that results in nonrandomized patients were similar to those in patients randomly assigned to a cooked diet gives us some confidence that our results are not a function of selection bias. It may also be difficult to generalize our results to patients treated outside a PE or to patients not administered, as were our patients, antifungal prophylaxis with itraconazole, voriconazole, or lipid amphotericin. Nonetheless, at the least, our results suggest that a randomized trial similar to ours but conducted outside a PE would be appropriate. This is particularly the case as induction therapy changes in older patients to emphasize targeted rather than intensive therapy.

\section{AUTHORS' DISCLOSURES OF POTENTIAL CONFLICTS OF INTEREST}

The author(s) indicated no potential conflicts of interest.

\section{AUTHOR CONTRIBUTIONS}

Conception and design: Alison Gardner, Elihu Estey

Provision of study materials or patients: Alison Gardner, Stefan Faderl, Gautam Borthakur, Guillermo Garcia-Manero

Collection and assembly of data: Alison Gardner

Data analysis and interpretation: Alison Gardner, Gloria Mattiuzzi, Sherry Pierce, Mark Brandt, Elihu Estey

Manuscript writing: Alison Gardner, Elihu Estey

Final approval of manuscript: Alison Gardner, Gloria Mattiuzzi, Stefan Faderl, Gautam Borthakur, Guillermo Garcia-Manero, Sherry Pierce, Mark Brandt, Elihu Estey

\section{REFERENCES}

1. Bodey GP, Buckley M, Sathe YS, et al: Quantitative relationship between circulating leukocytes and infection in patients with acute leukemia. Ann Intern Med 64:328-340, 1966

2. Casewell M, Phillips I: Food as a source of Klebsiella species for colonization and infection of intensive care patients. J Clin Pathol 31:845-849, 1978

3. Wright C, Kominoa SD, Yee RB: Enterobacteriaceae and Pseudomonas aeruginosa recovered from vegetable salads. Appl Environ Microbiol 31: 453-454, 1976

4. Smith LH, Besser SG: Dietary restrictions for patients with neutropenia: A survey of institutional practices. Oncol Nurs Forum 27:515-520, 2000
5. Wilson BJ: Dietary recommendations for neutropenic patients. Semin Oncol Nurs 18:44-49, 2002

6. DeMille $D$, Deming $P$, Lupinacci $P$, et al: The effect of the neutropenic diet in the outpatient setting: A pilot study. Oncol Nurs Forum 33:337343,2006

7. Moody K, Finlay J, Mancuso C, et al: Feasibility and safety of a pilot randomized trial of infection rate: Neutropenic diet versus standard food safety guidelines. J Pediatr Hematol Oncol 28:126-133, 2006

8. van Tiel $F$, Harbers $M$, Terporten $P$, et al: Normal hospital and low-bacterial diet in patients with cytopenia after intensive chemotherapy for hematological malignancy: A study of safety. Ann Oncol 18:1080-1084, 2007

9. Estey E, Smith TL, Keating MJ, et al: Prediction of survival during induction therapy in patients with newly diagnosed acute myeloblastic leukemia. Leukemia 3:257-263, 1989

10. Thall PF, Simon R, Estey EH: New statistical strategy for monitoring safety and efficacy in singlearm clinical trials. J Clin Oncol 14:296-303, 1996

11. Thall PF, Sung HG: Some extensions and applications of a Bayesian strategy for monitoring multiple outcomes in clinical trials. Stat Med 17: 1563-1580, 1998

12. Goodman S: Toward evidence-based medical statistics. 2: The Bayes factor. Ann Intern Med 130:1005-1013, 1999

13. Mattiuzzi G, Estey $E$, Hernandez M, et al: Voriconazole and liposomal amphotericin B (Ambisome) effectively prevent mold infections in patients with acute myelogenous leukemia following remission induction chemotherapy. Blood 106:2773, 2005 (abstr) 